\title{
CÓMO IMPLEMENTAR UN DISEÑO CURRICULAR BASADO EN COMPETENCIAS
}

\author{
Nancy Dalida Martínez Barragán*
}

\section{RESUMEN}

El diseño curricular basado en competencias es una forma de organización académica que acerca la educación superior a las necesidades del entorno global. Los ambientes laborales, profesionales y sociales son cada vez más complejos, por lo cual requieren personas más competentes ante los nuevos paradigmas de la sociedad de la información, con economías basadas en gestión y construcción del conocimiento. El desarrollo de competencias genéricas - emprendimiento, gestión de recursos, trabajo en equipo, gestión de información, liderazgo, responsabilidad ambiental y manejo de la segunda lengua - implica un amplio dominio de desempeño. Tales competencias se acompañan de las específicas, orientadas al campo laboral, en un contexto educativo, investigador y propositivo que se planifica desde el currículo. La investigación realizada permitió la proyección del currículo con un marco especifico de competencias, las cuales, según Tuning, para América Latina, y el Ministerio de Educación Nacional, para Colombia, se clasifican en Instrumentales, Interpersonales y Sistémicas, a la vez que se adoptan como transversalidades que posibilitan avanzar desde el conocimiento universal al particular, creando nuevas formas, objetos de conocimiento y miradas complejas de la realidad.

\section{PALABRAS CLAVE}

Competencias, currículo, diseño curricular, investigación, pensamiento crítico, transversalidad.

\begin{abstract}
The competency-based curriculum is a form of academic organization that brings Superior to the needs of the global social environment education, workplaces, and professionals are increasingly complex and require more competent persons to new paradigms of information society with construction based on knowledge management and economies. The development of generic skills is a broad domain of performance; includes
\end{abstract}

* Maestría Dirección Estratégica, Planeación y control de la Gestiòn-Instituto Eurotechnology Empresas (Coruña) España 2011,Magister en Educación E. Learning y redes sociales. Universidad Internacional de la Rioja (España) 2014. Especialista en educación y orientación. Universidad Manuela Beltrán 1995. Licenciada en Ciencias de la Educación con Especialidad en Administración. Universidad INCCA de Colombia 1985. Docente de cátedras Virtuales y presenciales. Asesora en Pedagogía y Currículo Escuela Colombiana de Carreras Industriales ECCI. Unidad de Apoyo al Docente. dalimart2@yahoo.com,nmartinezb@ecci.edu.co 
entrepreneurship, resource management, teamwork, information management, leadership, environmental responsibility and management of the second language; these skills are developed along with specific workplace-oriented research on a planned and purposeful educational context from the curriculum. With the research developed it came to curriculum planning with a specific framework of competencies, which since Tuning for Latin America and the Ministry of Education in Colombia are you classified Instrumental, Interpersonal and Systemic, are adopted as transversalities that advance from knowledge universal to the particular, creating new forms and objects of knowledge, and complex views of reality.

\section{KEYWORDS}

Competencies, critical thinking, curriculum, mainstreaming, research.

\section{INTRODUCCIÓN}

El diseño de estructuras curriculares es un proceso complejo; la estructura debe satisfacer el alcance de saberes, el abordaje de contenidos y el desarrollo de competencias. Se logra mediante un proceso desarrollado al interior de la Institución el cual incluye la planeación de la mejora, el diseño o rediseño curricular, los procedimientos de ajuste y dosificación, la construcción del currículo en sí, su implementación y los criterios de evaluación tanto para el estudiante como para el mismo proceso de implementación con el fin de medir los resultados alcanzados.

En este sentido, también es necesario considerar su flexibilidad y secuenciación en cada uno de los niveles educativos y su apropiación en coherencia con la malla curricular. El diseño curricular tiene que ver con la forma pedagógica de llevar la teoría a la práctica y este proceso debe ser observable y medible con el fin de hacer los seguimientos o mantenimiento necesarios para realizar ajustes que propicien el éxito en la implementación.

La praxis pedagógica propicia espacios para comprobar que las distintas estrategias aplicables a la formación y el aprendizaje no dan los mismos re- sultados en los diferentes grupos porque especificidad varía; es decir, la práctica no surte el mismo efecto en todas las circunstancias. Una buena teoría se aplica a situaciones y contextos distintos, pero su resultado no es el mismo en cada uno de estos, lo cual forma parte de la experiencia académicopedagógica permitiendo al docente crear, pensary repensar su propia acción.

El docente de Educación superior, por tanto debe estar abierto al cambio, a diseñar prácticas acordes a las características del estudiante y los grupos, debe estar en capacidad de diseñar estrategias, metodologías y sistemas de evaluación que salgan del paradigma de lo puramente conceptual - memorístico, para abordar el aprendizaje significativo el cual según Ausubel (1976, p. 82) puede relacionarse con la efectividad de promover el cambio cognitivo significativo para la individualidad del estudiante y lo social. Tal Aprendizaje se logra mediante la combinación de los saberes que ya tiene el educando posee con los nuevos. Para ello el aprendizaje debe ser realista y científicamente viable de tal manera que favorezca el desarrollo de competencias en el estudiante. Estas dosificadas y propuestas desde el currículo dimensionan estudian- 
tes capaces de generar soluciones a problemas en entornos diversos; en esa medida cobraría valor el concepto: cuando es aplicado por el estudiante ${ }^{2}$ (Moreira 2000, p.2)

La gestión del currículo proporciona un ambiente de avance en el conocimiento siendo el docente mediador de aprendizaje con un desarrollo curricular continuo, interdisciplinar, que propicie la investigación y promueva el desarrollo de personas emprendedoras y comprometidas con la sociedad; con un marco amplio de competencias instrumentales, interpersonales y sistémicas conjugadas como un constructo subyacente que permita la internalización propuesta por Vygotsky (1987) propiciando un desarrollo cognitivo en referencia al contexto histórico, cultural en el que ocurre .

El diseño de currículo implica un cambio de paradigma educativo con participación de los actores mencionados. En dicho proceso incorpora las visiones para lograr implantar un imaginario en la práctica docente que desarrolle un modelo innovador. De esta manera se logra, que el modelo pedagógico sea una construcción y apropiación de la propuesta curricular. Para ello, también es importante centrar estrategias curriculares desde el punto de vista de la investigación pedagógica; es decir, investigar el propio accionar docente y su gestión curricular de manera que se propicien cambios pensados, la mejora continúa tanto a nivel estructural, como a nivel de la gestión Institucional en pro de la consecución de metas y objetivos contemplados en los PEl; todo ello en relación de pertinencia con los requerimientos locales y globales buscando impactos reales desde la relación dialéctica estudiantedocente-problema social.

\section{Marco teórico.}

Las Instituciones de educación Superior han trabajado multiplicidad de modelos para la formación profesional; Marín (1997, p. 97) menciona modelos tales como:

- Modelo articulado de universidad, estructura social, mediada por prácticas profesionales emergentes para satisfacer grupos societarios.

- Modelo centrado en la formación crítica articulado alrededor de una acción problematizadora y vinculada con teoría y práctica.

- Modelo centrado en la práctica profesional.

- Modelo centrado en la interdisciplinariedad y modular, vinculando desarrollo, investigación y servicio.

- Modelo educativo basado en una formación de calidad.

De esta revisión se desprende que los aspectos enunciados en las tendencias se quedaron en meros discursos y no impactaron la realidad del aula, menos aún la de la sociedad.

Hoy día se conocen algunos otros modelos que aún no han sido evaluados, pero que se encuentran en nuestras instituciones.

- Modelo curricular integrado en macro asignaturas, en objetivos comunes en un área de conocimiento, idóneos para el desarrollo de habilidades y destrezas para la solución de problemas, con una organización curricular alrededor de temas procedentes del campo profesional.

- Modelo dirigido a la resolución de problemas y nucleado por estudios de casos.

2 "Ausubel (1978, p.86) define conceptos como "objetos, eventos, situaciones o propiedades que poseen atributos criteriales comunes y se designan, en una cultura dada, por algún signo (...) aceptado" (Moreira, 2000 a, pág. 21). 
- Modelo basado en los valores sociales y humanísticos, en el trabajo en equipo e investigación. También los modelos de formación profesional pueden analizarse desde la fundamentación epistemológica, en donde la formación profesional parte de una racionalidad técnica propia del positivismo versus un paradigma comprensivo diferente al paradigma clásico

- Modelos basados en taxonomía de objetivos

Es por ello que para consolidar una propuesta pedagógica o un modelo educativo hay que hacernos preguntas sobre nuestras prácticas pedagógicas en torno a temas como:

- El currículo y su diseño

- El desarrollo del currículo y la evaluación

- El proceso enseñanza aprendizaje

- El papel del docente y el tutor virtual

- La relación entre docente y estudiante

- Los fines y estrategias que requiere nuestro contexto

- Las competencias que requieren hoy nuestros estudiantes y docentes

- La coherencia entre el saber y el hacer

- La teoría pedagógica y la práctica que subyace desde la docencia

En el campo de la Educación Superior tenemos experiencia directa e indirecta de gran cantidad de procesos de cambio, reformas, nuevas políticas educativas que implican reorientar las formas de enseñanza y nuevas metas educacionales; sin embargo casi nunca nos detenemos a pensar en la complejidad; es decir para iniciar un proceso de cambio hay que tomar una actitud analítica, conciliadora y holística que perciba, registre y analice las características de:

- Los docentes

- Los estudiantes

- El sistema
- El contexto

- Los factores internos y externos

El desarrollo del Currículo, en general debe posibilitar:

1. Criterios de selección de los contenidos o propuesta de saberes (conceptos básicos disciplinares): ¿Qué es lo que debe aprenderse y enseñarse?

2. Principios para la estrategia de enseñanza: ¿Cómo debe aprenderse y enseñarse?

3. Criterios de secuencia y/o dosificación de los contenidos, procesos y proyectos: ¿Cómo apropiar contenidos significativos?

4. Principios para diagnosticar puntos fuertes y débiles del estudiante: ¿Cómo propiciar la evaluación diagnóstica, los planes de mejoramiento y realizar seguimiento al avance del estudiante?

5. Criterios de Flexibilización e internacionalización: ¿Cuáles son los contenidos significativos según los requerimientos para el profesional actual?

6. Criterios de evaluación: ¿Cómo desarrollar un sistema de evaluación pertinente al diseño curricular

7. Definición y Estructura de las competencias: ¿Cuáles con los componentes de la competencia para la Institución?

8. Sistema de seguimiento a la implementación con el fin de determinar avances y fortalezas y detectar debilidades y mejoras: ¿Cómo está diseñado en Sistema de seguimiento a la implementación curricular? 
El currículo se hace realidad a través de las funciones de la Educación Superior, tomando en consideración los ámbitos ecológico, internacional, socio cultural, económico y político. Con una visión global se propician las condiciones para la formación de ciudadanos profesionales, capaces de proponer nuevos modelos de desarrollo y nuevas maneras de construir los conocimientos.

En la figura 1 se puede observar la relación del currículo con factores que lo integran.

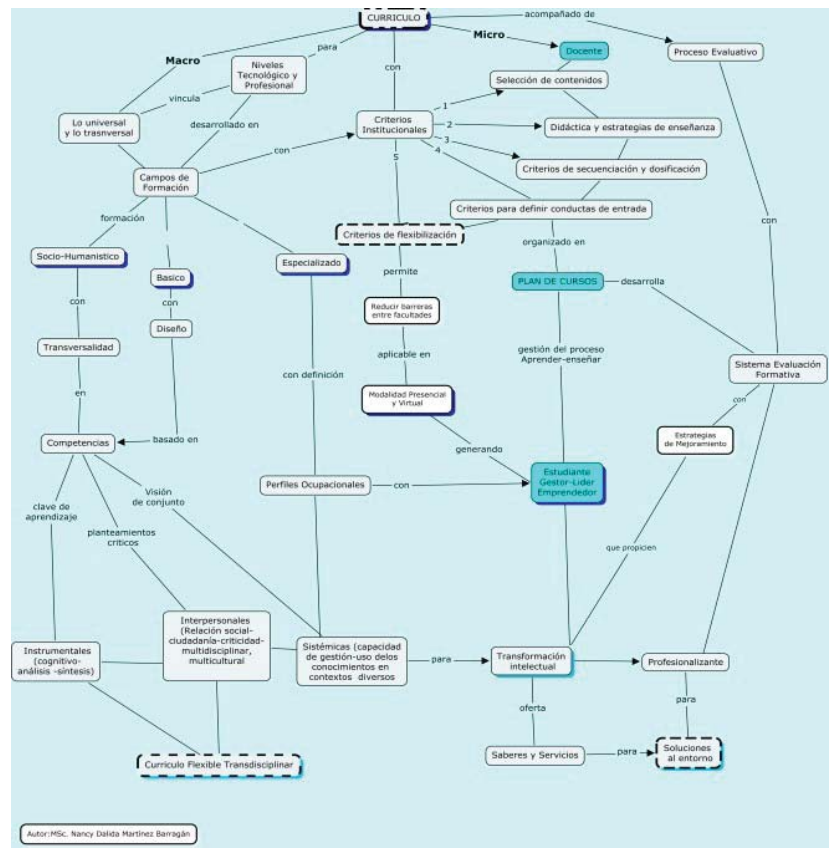

Figura 1. Currículo, Competencias y factores que lo integran Fuente: elaboración propia.

1.1 Flexibilidad curricular: La flexibilidad en educación superior está relacionada con tres aspectos: ingreso, proceso y perfil. De acuerdo con la política pública de educación superior por ciclos y competencias, la flexibilidad en el ingreso implica permitir ingreso a diversidad de estudiantes. La flexibilidad en el proceso requiere participación del estudiante en selección de su propio itinerario según sus objetivos propios y los objetivos del programa. Y la flexibilidad en el perfil implica que la Institución tenga claro el perfil que espera del egresado. La flexibilidad por tanto debe garantizar la movilidad del estudiante entre Instituciones, programas, niveles de formación y modalidades presencial y virtual. (Colombia, MEN. 2007, p. 23)

La flexibilidad implica además que la Institución sea generadora y formadora de personas íntegras, con competencias que les permitan movilidad y articulación con el entorno laboral.

En ese sentido es necesario mencionar "algunas tendencias en el mundo del trabajo que afectan significativamente los criterios de definición y diseño curricular, en particular aquellos que privilegian la importancia de los perfiles ocupacionales, para la previsión de desempeños ocupacionales específicos" (Celis y Gómez 2005, p. 2)

En síntesis, la flexibilidad curricular puede definirse en términos de: estructura, paso de un ciclo o nivel a otro, en relación con sistema de créditos y rutas para el estudiante o como cualidad trascendente del egresado para su inmersión en contextos variados, donde aplicará las competencias desarrolladas en la Educación Superior en la resolución de problemas y la generación de proyectos.

1.2 Transversalidad: El término y transversalidad está referido a aquello que atraviesa o se visualiza de un lado a otro. La Declaración Mundial sobre la educación en el siglo XXI (Cátedra Unesco, 2000) establece como función y misión de la Universidad la educación, la formación y la investigación; así como las funciones éticas de autonomía, responsabilidad para dar respuesta a las necesidades presentes y futuras de la sociedad.

La transversalidad curricular se centra en aquellos aspectos o componentes que la Institución educativa considera relevantes en la formación de los profesionales y establece encuentro entre los saberes disciplinares de manera que se conjuga la universal y lo especifico. 
Esta transversalidad implica una mirada holística ya que, cuando se aplica al plan curricular se definen el conocimiento y las competencias que se espera desarrollar en los estudiantes. Por ende la entidad está llamada a promover transformaciones y asumir nuevos paradigmas, teniendo en cuenta que los ejes transversos pueden definirse desde contenidos, valores, habilidades, campos de formación y de conocimiento, y competencias, entre otros aspectos.

Por tanto el Currículo es un ámbito compuesto con elementos interrelacionados que requiere unas etapas planificadas e intencionadas desde lo administrativo y lo académico para su implementación.

"En términos más concretos, el currículo es un espacio, una propuesta de enlace entre la teoría y la práctica educativa, determinado por factores políticos, culturales, económicos y sociales" (García, 2008, p. 13). En consecuencia la flexibilidad tiene que ver, también, como reconocimiento de la diversidad, un ente adaptable y diversificado. De ahí que un Currículo con transversalidades definidas permitiría igualdad de oportunidades y un equilibrio entre una educación superior que, sin perder su visión de formación integral del estudiante, prepare para un futuro productivo generando profesionales críticos, con capacidades y competencias para intervenir en el proceso de desarrollo social.

1.3 Pertinencia: Por pertinencia se entiende lo que es necesario y oportuno. Está relacionada con el aporte de la educación superior al entorno económico.

Educación pertinente es aquella que está en condiciones de contribuir a la transformación y desarrollo de las comunidades locales y nacionales, preparar para la inserción en el mundo del trabajo en la medida en que se articula con el sector productivo y aportar a la construcción de un mundo más justo, más equitativo y comprometido con el ambiente. Por consiguiente el currículo y los métodos de enseñanza deben ser flexibles para adaptarse además a las necesidades educativas del estudiante y a los diversos contextos, de forma que el estudiante apropie los contenidos de manera significativa para constituirse sujeto social y gestor de soluciones y transformaciones en contextos variables

La pertinencia también puede ser entendida desde la organización del currículo "Los componentes y los cursos no deben entenderse como un listado deContenidos separados y yuxtapuestos" (Díaz, 1995, p. 85), sino como componentes de campos de conocimiento general teórico- prácticos, que aplican métodos y didácticas para generar ambientes de aprendizaje que propongan experiencias significativas de aprendizaje.

1.4 Equidad: La equidad es un concepto estrechamente articulado con los principios de justicia e igualdad, un estado en el cual se reconoce a cada persona como igual, que implica la no distinción de personas ni grupos, implica un tratamiento imparcial de otorgamiento de los que cada individuo merece.

La equidad comprende, además, apoyo a las personas más necesitadas, en especial, ofreciendo ayuda a los estudiantes para que logren los objetivos educativos de manera equiparable.

1.5 Calidad: La Calidad educativa se refiere al avance hacia la mejora continua; está orientada a la excelencia y es relevante para cada ciclo educativo en la medida que defina requerimientos, identifique escenarios futuros y exigencias del entorno globalizado. Todo ello con el fin de propiciar la gestión del conocimiento, en una propuesta de aprendizaje y desarrollo de competencias, es decir, con un currículo orientado a la internacionalización de un sujeto competitivo, con habilidades de gestión ambiental y de recursos, responsabilidad social, capacidad crítico-analítica e innovadora.Adicionalmente la calidad implica además la posibilidad de dar cuenta del avance del estudiante en relación con los resultados del aprendizaje (estándares, pruebas censales, comparativos con índices Nacionales e Internacionales) 
y también se manifiesta como accionar institucional hacia las metas con liderazgo profesional desde las funciones de la docencia investigación y proyección social.

El concepto de calidad tiene que ver con la necesidad de asegurar al estudiante el desarrollo de competencias independientemente de sus características individuales o socioeconómicas, para participar en la vida social y productiva en las mismas condiciones (Colombia MEN. 2007, p. 49)

1.6 Investigación: La educación Superior en Colombia requiere el desarrollo de competencias en pensamiento crítico, analítico, innovación y desarrollo de pensamiento complejo que permitan realmente producir soluciones reales a los problemas actuales. La experiencia demuestra que la educación se queda en el inicio de la acción investigativa y apenas alcanza los primeros niveles del proceso, en la producción primaria de datos y la producción de proyectos de grado que distan mucho del abordaje del conocimiento conducente a ver la Institución de educación superior como parte de una amplia red de Instituciones de conocimiento que promuevan la Investigación-acción.

Investigar implica un proceso que interrelacione el objeto de conocimiento, con la gestión de éste y los métodos de indagación, de una manera transversal desde el currículo de cualquier asignatura e involucrando situaciones problémicas reales con el fin producir proyectos, soluciones que impacten socialmente.

1.7 Pensamiento crítico: La capacidad humana de analizar, incorporar, reelaborar, reflexionar y conformar juicios con criterio propio, es lo que se denomina como pensamiento crítico. Es el razonamiento que se hace claridad, precisión, equidad y proyección de las capacidades intelectuales, con el fin de alcanzar una posición reflexiva y justificada sobre un tema.
El pensamiento crítico y analítico es una competencia que emerge del aprendizaje significativo al complejo y que permite que el estudiante:

- Plantee preguntas y formule problemas en forma clara

- Compile, organice y seleccione información de fuentes diversas

- Concluya en forma razonada sobre situaciones concretas disciplinares e interdisciplinares

- Explique problemas en forma razonada y proponga alternativas de solución

- Interactúe con otras disciplinas y saberes demostrando conciencia de las implicaciones de su pensamiento

- Aplique inteligencia emocional en la resolución de problemas

- Distinga implicaciones y consecuencias de decisiones

1.8 Creatividad e innovación: Algunas autores proponen definiciones del concepto de innovación:

Innovación en un sentido económico consiste en la consolidación de un nuevo producto, proceso o sistema mejorado. (Freeman, C, 1982, citado por Medina Salgado y Espinosa 1994 en Innovación y creatividad, wiki libro)

La innovación es la herramienta específica de los empresarios innovadores; el medio de explotación del cambio como una oportunidad para un negocio diferente (...) hasta que el hombre encuentra la aplicación de algo natural y entonces lo dota de valor económico. (Drucker, 1985, p. 67).

En estas definiciones como en otras existen coincidencias en la ideología de cambio, de lo novedoso, que tenga éxito en el mercado. El cambio estaría en el producto o servicio que se oferta.

Desde el punto de vista de la Investigación la capacidad innovadora se propicia desde las competencias 
transversales dado que la Innovación es producto del conocimiento adquirido interdisciplinarmente, las habilidades y capacidades desarrolladas a través de la acción educativa y del desarrollo de las potencialidades del estudiante.

En efecto, para evidenciar esta capacidad Innovadora el estudiante aportará proyectos, investigaciones y acciones emprendedoras que impacten los distintos sectores de la economía nacional y se proyecten al entorno global desde el desarrollo curricular mismo.

En la figura 2 se puede observar la relación entre la investigación y la innovación como aquí se concibe.

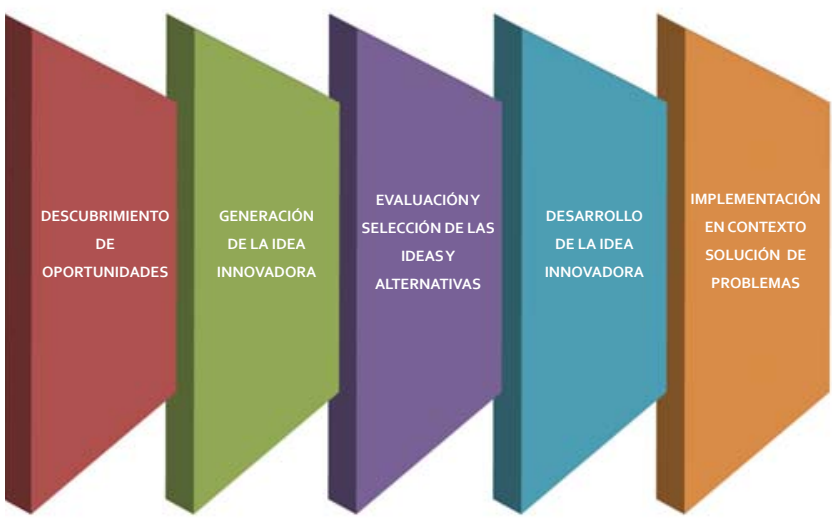

Figura 2. Investigación e innovación Fuente: elaboración propia.

1.9 Competencia: La competencia, para efectos de este documento, es una combinación compleja de conocimientos, desarrollos, habilidades, conceptualizaciones aplicadas, actitudes, valores, habilidades para desempeñarse en forma competitiva en contextos y situaciones diversas.

Las competencias pueden consistir en motivos, rasgos de carácter, conceptos de uno mismo, actitudes o valores, contenido de conocimientos, o capacidades cognoscitivas o de conducta aplicable en entornos y situaciones diversas.

Se puede asumir que una persona demuestra una competencia, cuando existan características que se puedan medir (desde estándares fiables) y cuya presencia se pueda demostrar de una manera significativa; es decir cuando se evalúan los desempeños de competencia, las conductas, comportamientos, procesos y productos observables que produce el sujeto de aprendizaje.

1.10 Clasificación: La clasificación de Competencias se apropia desde Proyecto Tuning para América Latina (Beneitone, et al 2004-2007) y Ministerio de Educación para Colombia (2007) tomando como base: "Una vez definidas las competencias comunes, cada institución de educación superior (IES) puede abordarlas de acuerdo con su estructura curricular, de manera que se trabajen pertinentemente y con plena autonomía4 "las competencias se clasifican desde los genérico constituyéndose en un referente macro para el desarrollo curricular, enuncian las competencias generales que todo profesional debería desarrollar a través de su proceso educativo y se clasifican en: Instrumentales, Interpersonales y Sistémicas proponiendo así un amplio marco de desarrollo de habilidades, destrezas, comportamientos, aplicación de conceptos, se sale del paradigma de que el estudiante para demostrar una competencia, se limita a seguir Instrucciones; para pasar a una toma de decisiones basada en el conocimiento y dar aplicabilidad al mismo como agente de cambio en la sociedad

Una competencia debe poder evaluarse a través de desempeños, aplicaciones, acciones propias o de equipos, proyectos, creaciones e innovaciones (lo que comúnmente se denomina evidencias de desempeño) y se plantea o redacta como capacidad, habilidad, destreza o actitud desarrollada, la creatividad 
e innovación, elementos tales que la Institución de Educación Superior apropia a su contexto y especificidad en el modelo evaluativo.

\section{Métodos de investigación}

Durante el primero y segundo periodo académico de 2012 se desarrolló un proceso diagnóstico y estudio del estado del arte de los Currículos de las Asignaturas del Campo Socio-Humanístico de la Universidad $\mathrm{ECCl}$ se aplicaron los métodos: de observación directa participante, el analógico Comparativo y cuantitativo.

Se realizó el diagnóstico del estado de los microcurrículos, como aparece en la tabla 1 y la figura 3. Se dialogó luego con los docentes de los comités curriculares en talleres de apropiación de conceptos sobre Modelo Pedagógico, Competencias, transversalidad e interdisciplinariedad curricular; así como las competencias de investigación como eje del proceso educativo.

Una vez culminado este proceso, se dio comienzo a la divulgación del diseño curricular basado en competencias como despliegue del "Modelo Pedagógico Crítico-Social y Significativo" (Martínez y Veloza, 2012, p. 65) adoptado para la Universidad ECCI. Del mismo modo se desarrolló el proceso con cada Coordinación de programa para la incorporación de las competencias transversales Socio-humanísticas, definidas en la Matriz transversal en los Microcurrículos, como ejercicio de mejora curricular y avance al desarrollo del modelo.

Tabla 1. Microcurrículos revisados y retroalimentados

MICROCURRICULOS REVISADOSY RETROALIMENTADOS

\begin{tabular}{|l|c|}
\hline 2012.2 Y 2013.1 & \\
\hline Socio- Humanisticas / Investigación & 24 \\
\hline Monitoreo por programa Tecnológico & 40 \\
\hline Monitoreo por programa profesional & 36 \\
\hline Microcurrículos revisados & 96 \\
\hline
\end{tabular}

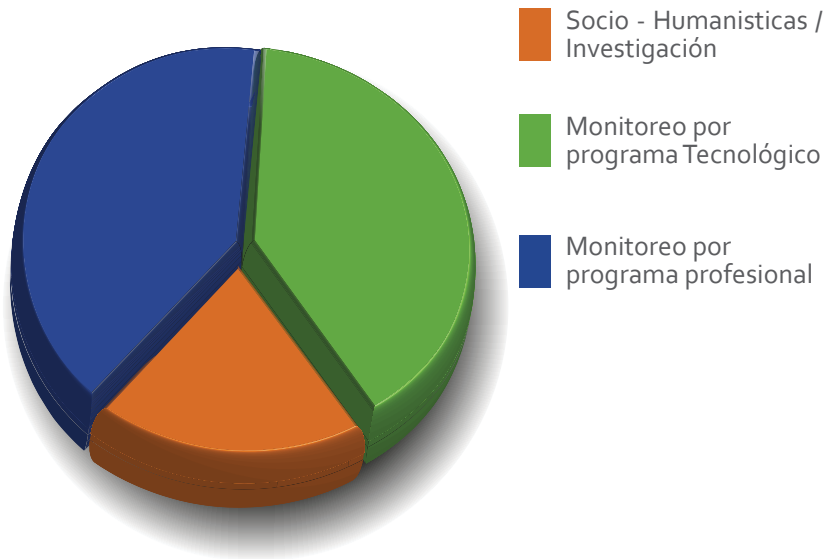

Figura 3. Micro currículos revisados

Fuente: elaboración propia.

El proceso implicó la revisión uno a uno de los Micro currículos y su ajuste al esquema y clasificación de competencias inicialmente para el campo transversal socio-humanístico como se observa en la figura 4. Posteriormente se comenzó a desarrollar la misma estructura de competencia para los Campos Básico y Especializado. Véanse también la tabla 2 y la figura 5.

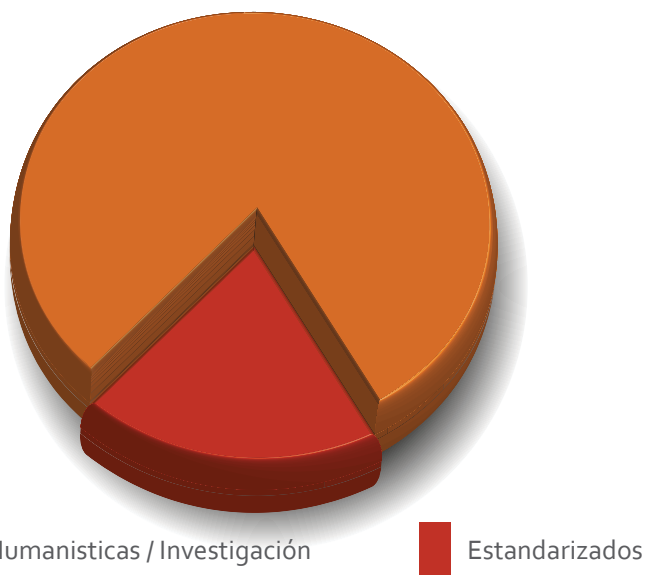

Socio - Humanisticas / Investigación

Figura 4. Microcurrículos estandarizados/contenidos temáticos. Identificación/nombre.

Fuente: elaboración propia.

Tabla 2. Monitoreo a microcurrículos

\begin{tabular}{|c|c|c|}
\hline \multicolumn{3}{|c|}{ MONITOREO A MICROCURRICULOS } \\
\hline \multicolumn{3}{|c|}{$2012.2 Y 2013.1$} \\
\hline NIVEL & TOTAL & REVISADOS \\
\hline Tecnológico & 146 & 40 \\
\hline \multirow[t]{2}{*}{ Profesional } & 200 & 36 \\
\hline & & 76 \\
\hline
\end{tabular}




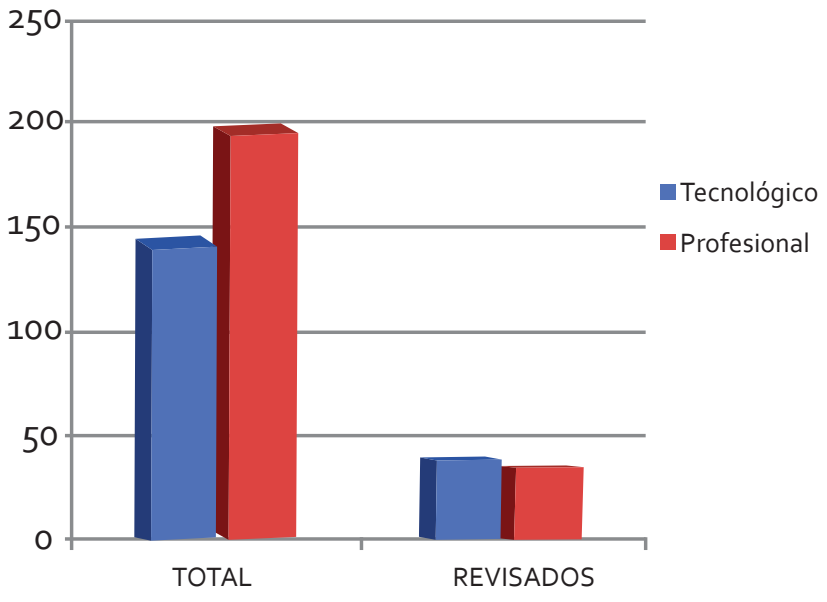

Figura 5. Microcurrículos monitoreados Fuente: elaboración propia.

La estructura de la competencia se definió con tres componentes, a partir de la investigación de referentes teóricos y las sugerencias dadas en la visita de pares académicos del Ministerio de Educación Nacional: acción, objeto y condición como se muestra en la figura 6 .

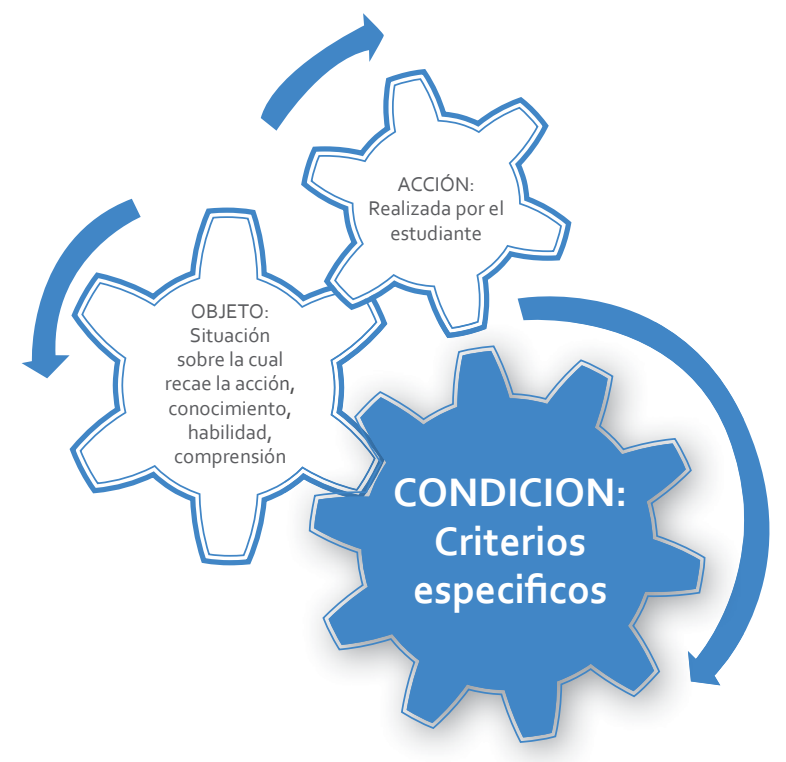

Figura 6. Estructura de la competencia

Fuente: elaboración propia.

Esta estructura se adopta y se codifica para el Campo Socio-Humanístico generando la matriz de Compe- tencias de dicho campo dosificada para los Niveles Tecnológico y profesional.

Durante el segundo de periodo 2012 en reuniones citadas por la dirección de currículo, con participación de líderes curriculares, se explicó y discutió el proceso de ajuste a competencias como despliegue del diseño curricular basado en competencias.

La tabla 3 y la figura 7 dan cuenta del número y tipo de asistentes a las reuniones del comité socio-humanístico. La tabla 4 y la gráfica 8, por su parte, muestran la participación del comité de ciencias básicas.

Tabla 3. Docentes y directivos participantes en reuniones curriculares 2012.2 Comité Socio-humanístico

\begin{tabular}{|c|c|c|}
\hline DOCENTES & DIRECTIVOS/COORDINADORES & TOTAL \\
\hline 20 & 16 & 36 \\
\hline
\end{tabular}

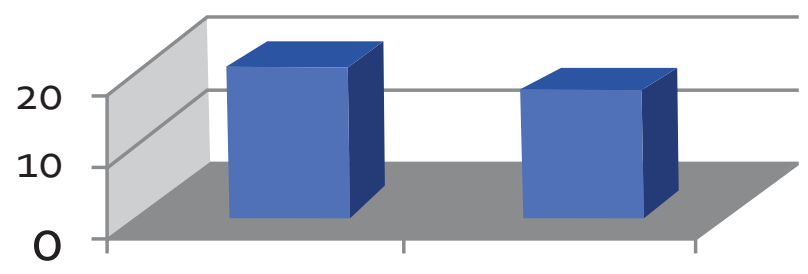

\section{DOCENTES DIRECTIVOS}

Figura 7. Docentes participantes reuniones curriculares 2012.2 Comité Socio-Humanístico

Fuente: elaboración propia.

Tabla 4. Docentes y directivos participantes en reuniones curriculares 2012.2 Comité de ciencias básicas.

\begin{tabular}{|c|c|}
\hline DOCENTES & COORDINADORES \\
\hline 16 & 6 \\
\hline
\end{tabular}




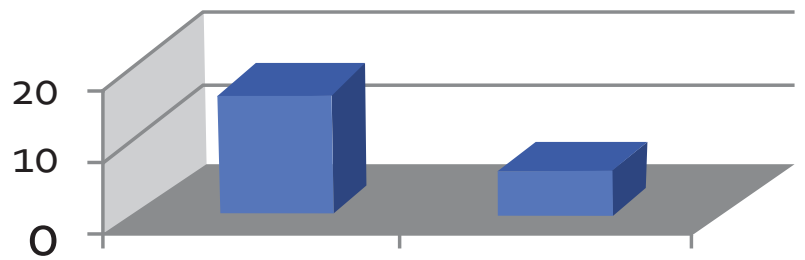

DOCENTES COORDINADORES

Figura 8. Docentes participantes Comité Ciencias Básicas Fuente: elaboración propia.

Durante el primer periodo de 2013 se continuó el proceso de asesorías en pedagogía y currículo para su implementación, desde la unidad de Apoyo al Docente con reuniones convocadas por programa. Se desarrollaron en total siete reuniones con asistentes docentes y líderes curriculares como se muestra en la tabla 5 .

Tabla 5. Asistentes a reuniones de grupo

\begin{tabular}{|c|c|}
\hline Reuniones & Asistentes \\
\hline 1 & 8 \\
\hline 2 & 10 \\
\hline 3 & 3 \\
\hline 4 & 4 \\
\hline 5 & 8 \\
\hline 6 & 4 \\
\hline 7 & 11 \\
\hline TOTAL & 48 \\
\hline
\end{tabular}

En el transcurso del segundo periodo 2013 se desarrolla asesoría personalizada para ajuste de los Micro currículos y asesorías grupales a solicitud de los coordinadores de programa. Ello, con el fin de generar el proceso de actualización general de los mismos a competencias, con miras a estandarizar su diseño en consonancia con el modelo pedagógico Institucional. La tabla 6 y la figura 9 permiten tomar nota del número de participantes en dichos encuentros. Por su parte la figura 10 muestra el comparativo de asistencia entre el primero y segundo semestres del año.
Tabla 6. Asistentes a reuniones de grupo

\begin{tabular}{|c|c|}
\hline Reuniones & Asistentes \\
\hline 8 & 2 \\
\hline 9 & 4 \\
\hline 10 & 5 \\
\hline 11 & 11 \\
\hline 12 & 12 \\
\hline 13 & 11 \\
\hline 14 & 32 \\
\hline TOTAL & 77 \\
\hline
\end{tabular}

\section{Total Atenciones Grupales 2013-2}

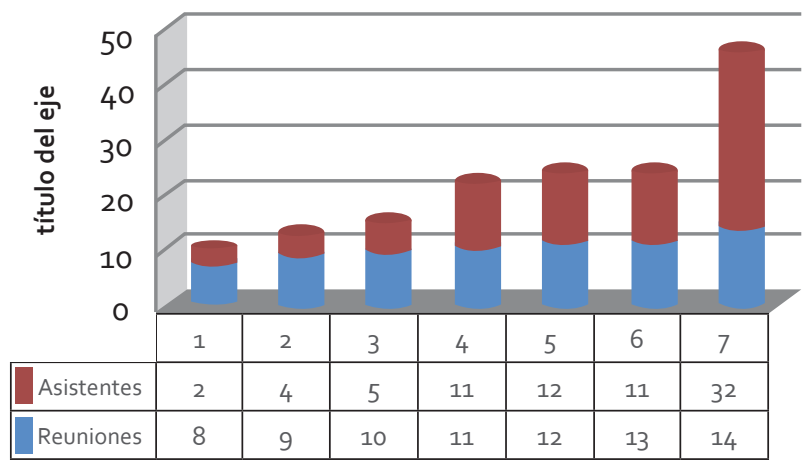

Figura 9. Asistentes a reuniones grupales Fuente: elaboración propia.

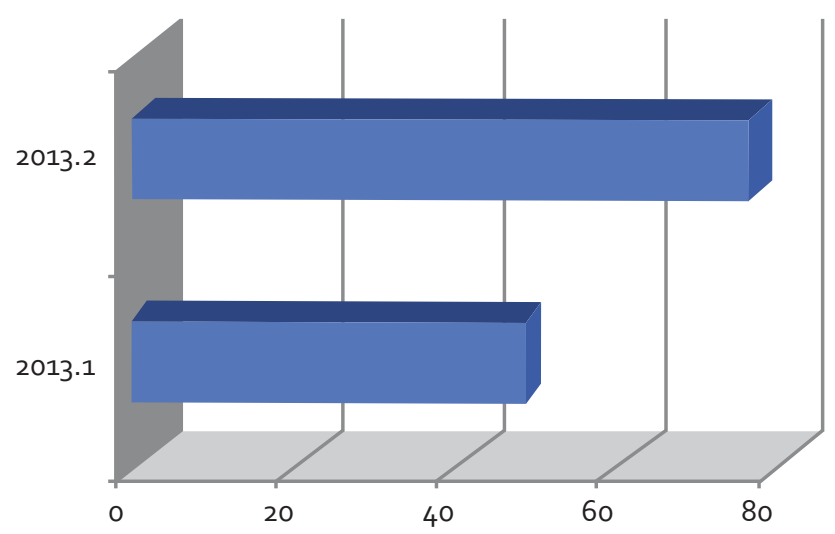

Figura 10. Comparativo asistencia a reuniones por coordinación Fuente: elaboración propia. 


\section{Resultados y discusión}

El proceso de análisis individual de la práctica pedagógica, detección de debilidades en la conceptualización curricular, metodológica, didáctica y del modelo pedagógico mismo; se dinamizó con la realización de los talleres-reunión desarrollados con coordinadores, docentes y líderes curriculares de los diferentes programas y facultades, eventos convocados por la Dirección de Currículo, durante el segundo periodo 2012 y el primer periodo 2013.

Posteriormente se dinamizó un segundo ciclo de reuniones -taller para convocar y discutir con una población alcanzada de 216 docentes y directivos (en atención grupal y personalizada) Mediante dicho proceso se aclararon y trabajaron los tópicos en falencia para brindar elementos claros y directos de apropiación del diseño curricular basado en competencias. Este se desplegó desde el Modelo Pedagógico, la política y la misional institucional hasta su aplicación a los microcurrículos. Así mismo, se impulsó el proceso de apropiación de las competencias transversales definidas como institucionales; luego se propició la aplicación de la misma estructura a las competencias de los campos de formación básico y especializado en la Escuela Colombiana de Carreras Industriales.

La metodología adoptada para la revisión de los micro currículos ha sido de gran ayuda a los docentes para avanzar en el despliegue del modelo pedagógico; sin embargo persiste una resistencia en algunos docentes y directivos para salir de paradigmas anteriores ,ya que el Modelo Pedagógico y el rediseño curricular que los traduzca al aula implican la adaptación a paradigmas educativos más complejos, con un compromiso al trabajo curricular innovador, con seguimiento permanente y rediseño al 100\%. Por tanto se presentaron condicionantes en la revisión sistemática, pues persiste el paradigma de que es más efectiva la educación basada en el contenido puramente disciplinar, que la educación que desarrolle una amplia gama de competencias en un modelo de connotación holística. Este no se queda en la simple formación por competencias (laborales o de cultura para el trabajo), sino que da el paso a una universidad transformadora de conocimiento, desde un diseño curricular con un amplio espectro de competencias.

Todo lo anterior es posible desde una gestión curricular de aula, en el marco de la cual se encuentre el compromiso de docentes de tiempo completo, cátedra, coordinadores y autoridades académicas, en una realización de la docencia desde la perspectiva crítica, transformadora y reconociendo al estudiante como centro del proceso de aprendizaje y su proceso formativo como integralidad humana

De acuerdo con lo expresado se propuso el Procedimiento de Revisión y estandarización del diseño curricular, a partir de la verificación de su actualización al diseño basado en competencias. Para ello se realizaron las siguientes actividades

- Elaboración de la propuesta por parte de los expertos disciplinares

- Revisión por parte de los líderes curriculares

- Envío para revisión y asesoría pedagógica

- Verificación de apropiación de competencias del Campo Socio-Humanístico

- Revisión de competencias de los Campos básico y especializado de acuerdo con la Estructura definida para la Institución

- Envío de comunicación y retroalimentación a la coordinación

- Verificación de cumplimiento del proceso

- Envío a Dirección de Currículo

- Aprobación final

\section{CONCLUSIONES}

El Currículo constituye un elemento clave para cualquier sistema educativo desarrollado a partir de un modelo pedagógico definido, en la medida en que permite materializar y aplicar las ideas y concepciones establecidas como base pedagógica; presenta dos aspectos diferenciados y al mismo tiempo in- 
terconectados: el diseño y la acción, lo estático y lo dinámico, el modelo y la realidad, que, aunque esenciales ambos para el desarrollo del aprendizaje intencional en las instituciones educativas, pueden aparecer desconectados e incluso aislados. Ello ha implicado, investigación, reflexión pedagógica y un redireccionamiento en la fase dinámica; que en cualquier diseño curricular debe incluirse, no solamente lo previsto, lo planificado, lo intencional, sino también las condiciones en las cuales se efectúa el proceso aprender-enseñar, y la influencia de dicho proceso sobre los educandos, teniendo en cuenta que el currículum se centra en el sistema educativo para el logro de los objetivos de aprendizaje y a la vez el desarrollo de competencias; por tanto un currículo o modelo basado en competencias debe definir desde su planificación las competencias genéricas o generales y específicas a desarrollar con los Pensums.

A partir de los resultados del proceso de investigación desarrollado se impulsa la gestión del modelo pedagógico y la alineación del diseño curricular, se proyecta la Educación Superior a una dimensión internacional mediante currículos flexibles, inter y transdisciplinares que desarrollen competencias desde programas académicos que permitan potenciar beneficios y afrontar retos con participación de docentes e investigadores en educación. El objetivo es cristalizar experiencias e iniciativas conjuntas que beneficien la formación de los profesionales críticoanalíticos, emprendedores, capaces de resolver problemas con pertinencia social.

El Currículo por consiguiente debe organizarse en forma estructurada, estar programado y diseñado según la Institución, sus principios y filosofía, su modelo formativo y la epistemología pedagógica adoptada. También debe propiciar la formación integral del estudiante y definir el tipo de persona que desea egresar y por ende propiciar la transformación de los entornos y contextos para evidenciar cambios y soluciones que desarrollen históricamente tanto al estudiante/profesional como al colectivo/ lo social.

\section{REFERENCIAS}

Ausubel, D. P. (1976). Psicología educativa. Un punto de vista cognoscitivo. Ed. Trillas. México

Moreira M. A. (2000) Aprendizaje significativo un concepto subyacente. Instituto de Física, UFRGS .Brasil. pg 2.

Vygotsky. (1987) El desarrollo de los procesos psicológicos superiores, teoría critica. Grijalbo, México, 123-140.

Marín (2007) La formación profesional disciplinaria: Vías alternas en el campo d los cambios, en Alba (Comp). El currículo Universitario, México, Centro de Estudios sobre la Universidad/Plaza y Valdez.

Celis, J y Gómez, V (2005) Factores de innovación curricular y académica en la educación superior. En: Revista ie Red: Revista Electrónica de la Red de Investigación Educativa [en línea].Vol.1, No.2 Disponible en Internet: <http://revista.iered.org>. ISSN 1794-8061

UNESCO.( 2000) Universitat de Girona . Convenio Internacional. Cátedra UNESCO Recuperado Marzo de 2014 de: http://www.catedraunesco.com/?p=la_ catedra\&lang=es

García. N. (2008) Currículo y flexibilidad curricular. Currículo sendero o territorio? Ponencia evento GlNIC. Universidad de Antioquia. Medellín. Colombia

Díaz B., F. (1995). Metodología del diseño curricular para la Educación Superior. Editorial Trillas, México. Fuentes, $\mathrm{H}$.

Ministerio de Educación Nacional. (2007) Política pública educación superior por ciclos y competencias. Bogotà. Colombia.

Salgado y Espinosa (1.994) Innovación y creatividad: Evolución del concepto de innovación. Wiki libro sección 1. Recuperado http://www.eoi.es/wiki/ index.php/Evoluci\%C3\%B3n_del_concepto_de_ Innovaci\%C3\%B3n_en_Innovaci\%C3\%B3n_y_creatividad_2 
Drucker, P (1985).The discipline of innovation .Harvard Bussines Review

Beneitone, P, Esquetini, C, et al. (2004-2007) Reflexiones y perspectivas de la Educación Superior en América Latina.

Ministerio de Educación Nacional. (2007) Definición de competencias genéricas. Bogotà Colombia Recuperado de: http://www.aspucol.org/14-ultimasnoticias/o9_may_12_competencias_genericas_min_ educacion.html

Martinez B .N, Veloza M. (2012) Documento Modelo pedagógico y Lineamientos Curriculares 2012. ECCI. Bogotà .Colombia

\section{BIBLIOGRAFÍA COMPLEMENTARIA}

Ausubel, D. P. (1973). "Algunos aspectos psicológicos de la estructura del conocimiento". En Elam, S. (Comp.)La educación y la estructura del conocimiento. Investigaciones sobre el proceso de aprendizaje y la naturaleza de las disciplinas que integran el currículum. Ed. El Ateneo. Buenos Aires. Págs. 211-239.

Ausubel, D. P. (1976). Psicología educativa. Un punto de vista cognoscitivo. Ed. Trillas. México

Buxarrais, M. R y otros. (1991). "L'interculturalisme en el currículum. El racisme", Barcelona: Rosa Sensat.

Corral, R. (2004). El currículo basado en competencias. Ponencia, Universidad

de La Habana. Cuba

Celis, Jorge y Gómez, Víctor. (Enero-Junio de 2005). Factores de innovación curricular y Académica en la educación superior. En: Revista ie Red: Revista Electrónica de la Red de Investigación Educativa [en línea].Vol.1, No.2 Disponible en Internet: <http://revista.iered.org>. ISSN 1794-806
Díaz Villa; M. (2002), Flexibilidad y Educación Superior en Colombia. Serie Calidad de la Educación Superior No. 2 ICFES - MEN Bogotá, D.C.

Díaz, M. (2002) La flexibilidad en la educación superior ICFES. Bogotá.

Díaz B., F. (1995). Metodología del diseño curricular para la Educación Superior. Editorial Trillas, México. Fuentes, $\mathrm{H}$.

Díaz, M. \& Gómez, V. M. (2003) La Formación por Ciclos en la Educación Superior. ICFES. Serie Calidad de la Educación Superior, No. 9, 2003.

Díaz B., A. (1992). Ensayos sobre la Problemática Curricular. Editorial Trillas, México.

Drucker, P.F. (1997). La Innovación y el empresariado innovador: la práctica y los principios. Clásicos del Management. Barcelona: Apóstrofe.

Eurydice (2002). Key competentes. A developing concept in general compulsory education. Brussels: European Comission.

Fuentes, H. (1995). Fundamentos didácticos para un proceso de enseñanza - aprendizaje participativo. CEES, Universidad de Oriente, Santiago de Cuba.

Garagorri, X. (2007). Currículo basado en competencias. Aproximación en estado de la cuestión. Aula de innovación educativa (XVI): 47-161

Gonzàlez M. V. Qué significa ser un profesional competente. Revista Iberoamericana de Educación. (ISSN: 1681-5653)

Hernández C y López J (2002) Disciplinas. Series de calidad en Educación Superior Na4. Bogotà D.C Icfes Horruitiner, P. (2000). El Modelo Curricular en la Educación Superior Cubana. Ponencia Central en 'Universidad 2000'. Editorial Félix Varela, La Habana. Martínez B Nancy, Veloza V Mauricio. (2012) Documento Lineamientos Curriculares y modelo pedagógico. ECCI. Bogotà. Colombia 
Mertens L. (2000). La gestión por Competencia laboral en la empresa y la formación profesional. OEI Recuperado de: http://www.oei.es/oeivirt/fp/iberfopo1. htm

Ministerio De Educación Nacional. (2007) Sistema de Aseguramiento de la calidad en la educación Superior. Bogotà. Colombia

Ministerio De Educación Nacional (2010) Decreto 1295

Moreira, M. A. (2000). Aprendizaje significativo crítico. Atas do III Encuentro Internacional de Aprendizaje Significativo. Peniche. Portugal, págs. 33/45. (Traducción de lleana Greca).

Pozo, J. I. (1989). Teorías cognitivas del aprendizaje. Ed. Morata. Madrid.

Salmi, J. (2001) La Educación Superior en un punto decisivo. En: Orozco, L. E. (Compilador). Educación Superior.

Segura, M. (2003) Perspectivas Teóricas para transformar la Gestión Docente en la Universidad de Carabobo. Tesis doctoral. Universidad de Carabobo. Mimeo.

Sierra, C. (2001). Modelo de acción- reflexión para la valoración del desempeño Profesional apoyado en la autoestima del docente de Educación Básica. Caracas: Universidad Santa María. Doctorado en Ciencias de la Educación.

UNESCO (1998) La Educación Superior en el Siglo XXI: Visión y Acción. Conferencia Mundial sobre la Educación Superior. París, 5 - 9 de Octubre.

Villaveces, Cardozo J. (2009) Competencia científica, tecnológica y gestión de la Información Recuperado Abril de 2014 de: http://www.mineducacion.gov. co/1621/article-189357.html
Zabalza M. A. (2007). Competencias Docentes del Profesorado Universitario. Calidad y desarrollo Profesional. Ed Narcea, SA. $2^{\text {a }}$ ed. $1^{\text {a }}$ reimpresión. Madrid, España.

Ministerio de Educación Nacional (2003) Diseño de Currículos por Competencias. Colombia. Recuperado de http://www.mineducacion.gov.co/1621/w3article-299212.html

Díaz, Villa M. Flexibilidad y educación Superior en Colombia Recuperado de. .http://hydra.icfes.gov.co/ esp/fomento/gcfom/pub/p_publica.html

http://www.blogger.com/blogger.g?blogID=3274270 356808189237\#allposts/postNum=1

Godoy .M Educación inicial. de:http://www.educacioninicial.com/ei/contenidos/oo/1450/1451.ASP

Careaga Butter. M. Curriculo basado en competencias de:http://marcelocareaga.blogspot.com/2008/04/ currculo-basado-en-competencias.html

http://educacion.ucv.cl/prontus_formacion/site/artic/20061220/asocfile/ASOCFILE120061220151232. pdf

Curriculo basado en competencias .Recuperado de:http://es.slideshare.net/almamaite/3-currculobasado-en-competencias

Christin. A Calidad educativa Recuperado de: http:// www.calidadeducativa.edusanluis.com.ar/

http://revista.iered.org>. ISSN 1794-8061

Ramírez .L (2009) Modelo pedagógico Critico-social Recuperado de: http://es.scribd.com/ doc/54372349/26/Modelo-Pedagogico-Critico-Social 